\title{
Minimum Action Path Theory Reveals the Details of Stochastic Transitions Out of Oscillatory States
}

\author{
Roberto de la Cruz \\ Centre de Recerca Matemàtica, Edifici C, Campus de Bellaterra, 08193 Bellaterra (Barcelona), Spain \\ and Departament de Matemàtiques, Universitat Autònoma de Barcelona, 08193 Bellaterra (Barcelona), Spain \\ Ruben Perez-Carrasco* and Pilar Guerrero \\ Department of Mathematics, University College London, Gower Street, London WC1E 6BT, United Kingdom \\ Tomas Alarcon \\ ICREA, Pg. Lluís Companys 23, 08010 Barcelona, Spain \\ Centre de Recerca Matemàtica, Edifici C, Campus de Bellaterra, 08193 Bellaterra (Barcelona), Spain \\ Departament de Matemàtiques, Universitat Autònoma de Barcelona, 08193 Bellaterra (Barcelona), Spain \\ and Barcelona Graduate School of Mathematics (BGSMath), Barcelona, Spain \\ Karen M. Page \\ Department of Mathematics, University College London, Gower Street, London WC1E 6BT, United Kingdom
}

(Received 21 April 2017; revised manuscript received 13 December 2017; published 19 March 2018)

Cell state determination is the outcome of intrinsically stochastic biochemical reactions. Transitions between such states are studied as noise-driven escape problems in the chemical species space. Escape can occur via multiple possible multidimensional paths, with probabilities depending nonlocally on the noise. Here we characterize the escape from an oscillatory biochemical state by minimizing the Freidlin-Wentzell action, deriving from it the stochastic spiral exit path from the limit cycle. We also use the minimized action to infer the escape time probability density function.

DOI: 10.1103/PhysRevLett.120.128102

Introduction.-Cells are intrinsically noisy. Such stochasticity arises not only from the production and degradation of cellular components, but also from their mutual interaction or even the interaction with other cells. Nevertheless, some cellular processes require a precise deterministic output, and noise-suppression mechanisms are necessary within the cell [1-4]. On the other hand, since fluctuations are an intrinsic component of cellular dynamics, mechanisms are in place that cells exploit to improve their function [3-8]. There exist situations in which randomness can even evolve predictably in constant environments [9].

Mean-field descriptions of biochemical processes can be analyzed using dynamical systems theory [10], where stable steady states, sustained oscillations, or even transients of the ordinary differential equations (ODEs) correspond to different possible cellular states $[11,12]$. Relevant examples including sustained oscillations are circadian rhythms [13-15], cAMP oscillations in Dictyostelium

Published by the American Physical Society under the terms of the Creative Commons Attribution 4.0 International license. Further distribution of this work must maintain attribution to the author(s) and the published article's title, journal citation, and DOI.
[16], cell-cycle regulation [17-19], or patterns of bursting in neuronal activity [20-24].

When molecular populations are small, the mean-field framework is inaccurate and a stochastic description is required. This involves the formulation of the master equation (ME) describing the underlying multivariate biochemical birth-death process [25]. Unfortunately the ME is rarely solvable analytically, requiring the use of Monte Carlo methods (such as the Gillespie algorithm [26]). These numerical methods are often computationally costly, even infeasible [27]. This is especially true in phenomena associated with rare fluctuations, as in the noise-induced escape from a basin of attraction in mean first passage time (MFPT) problems.

The analysis of MFPT problems started with rate theory, studying escape from stationary states in double-well potentials [28]. This theory was later extended to more complex systems (nonpotential, dissipative), making use of rare event theory and quasisteady state approximation [29-31]. Nevertheless, in spite of the importance of oscillatory phenomena in biology, most studies have tackled escape problems from point attractors, and a general theory of escape from stable limit cycles is lacking. In order to fill this gap, we consider two oscillatory models and unveil the utility of the 
minimum action path (MAP) method from large deviation theory $[32,33]$.

The models.-In order to study the limit cycle-fixed point transition we first consider a model based on the normal form of a subcritical Hopf bifurcation, showing that the method can be applied to multiple physical problems [34].

$$
\dot{r}=r\left(r^{2}-c^{2}\right) E(x, y), \quad \dot{\theta}=\omega .
$$

In particular, we have broken the symmetry of the normal form by introducing the elliptical term $E(x, y) \equiv$ $1-\left[\left(x-x_{c}-x_{0}\right)^{2} / a^{2}\right]-\left[\left(y-y_{c}\right)^{2} / b^{2}\right]=0$, where $(x, y)$ are the Cartesian coordinates and $(r, \theta)$ the polar coordinates with respect to the Cartesian point $\left(x_{c}, y_{c}\right)$. In this description, the stable fixed point is located at $\left(x_{c}, y_{c}\right)$ that is surrounded by a circular stable limit cycle of radius $c$ (Fig. 1 and the Fig. S1 in Supplemental Material [35]). The basins of attraction are separated by a limit cycle that will depend on $E(x, y)$ (for more details see Sec. I in Supplemental Material [35]). Hereinafter, this model will be referred to as the Hopf model.

We further consider a less prototypical model to explore the predictions of the MAP to study the firing of neurons using the Morris-Lecar model [23] (see Sec. II and Fig. S2 in Supplemental Material [35] which includes Ref. [36]). In this context, the coexistence of a stable point and a limit cycle, characteristic of type-II excitable behavior, gives rise

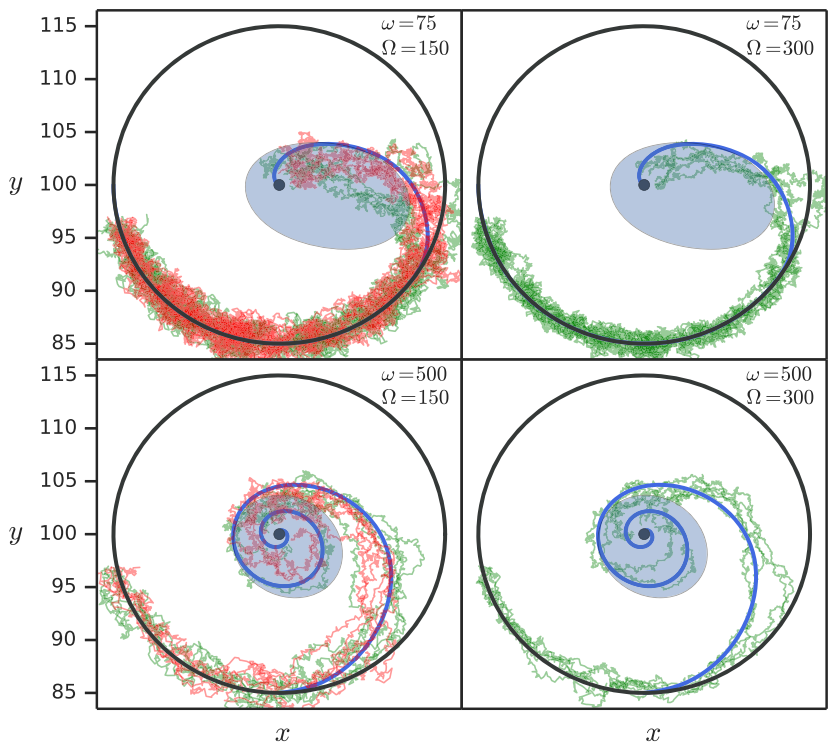

FIG. 1. Comparison of escape trajectories from the Hopf model limit cycle to the stable fixed point. Results show 5 trajectories of the CLE (green) compared with the MAP (blue) for different values of $\omega$ and $\Omega$. For $\Omega=150$, results are also compared with 5 Gillespie trajectories (red). The unstable limit cycle separating the basins of attraction (shaded area) is found by temporal inversion of Eq. (1). For the sake of clarity, only the last turn of each trajectory prior to escape is shown. The rest of the parameters are $x_{c}=100, y_{c}=100, x_{0}=5, a=8, b=5, c=15$. to stochastic spike trains. The properties of these spike trains are determined by the details of the stochastic jump out of the limit cycle. A detailed presentation of both models is given in the Supplemental Material [35], Sections SI and SII.

Stochastic description.-The details of the stochastic description will depend on the underlying process leading to the mean-field behavior given by Eq. (1). In the current Letter, we chose a compatible birth and death process by splitting the rhs of Eq. (1) into positive and negative contributions to the evolution of each species $\rho_{x}^{+}, \rho_{x}^{-}$, $\rho_{y}^{+}, \rho_{y}^{-}$, where the intensive magnitudes $x, y$ can be related to the number of elements of each species, $X=x \Omega$ and $Y=y \Omega$ (see Supplemental Material [35], Secs. SI and SII). As $\Omega$ grows, the intrinsic noise is reduced, recovering the mean-field limit Eq. (1) when $\Omega \rightarrow \infty$. For large (but finite) $\Omega$, the master equation can be approximated by the chemical Langevin equation (CLE) [37,38],

$$
\begin{aligned}
& \dot{x}=\rho_{x}^{+}-\rho_{x}^{-}+\Omega^{-1 / 2} \sqrt{\rho_{x}^{+}+\rho_{x}^{-}} \xi_{x}(t), \\
& \dot{y}=\rho_{y}^{+}-\rho_{y}^{-}+\Omega^{-1 / 2} \sqrt{\rho_{y}^{+}+\rho_{y}^{-}} \xi_{y}(t),
\end{aligned}
$$

where $\xi_{x}(t)$ and $\xi_{y}(t)$ are uncorrelated white Gaussian noises, of zero mean, and autocorrelation $\left\langle\xi_{x}(t) \xi_{x}\left(t^{\prime}\right)\right\rangle=$ $\left\langle\xi_{y}(t) \xi_{y}\left(t^{\prime}\right)\right\rangle=\delta\left(t-t^{\prime}\right)$. Equations (2) imply that tuning the value of $\Omega$ allows us to investigate the role of fluctuations in the transition between the stable limit cycle and the fixed point.

Minimum action path.-The intrinsic noise described in the previous section allows for transitions between the limit cycle and the fixed point. Such transitions can occur through many possible transient trajectories, $\varphi(x(t), y(t))$. Nevertheless, not all the transitions are equally probable. In particular, for reaction systems, unlikely transitions decay exponentially with $\Omega, \mathcal{P} \sim e^{-\Omega \mathscr{S}(\varphi)}$ [39], where the decay rate $\mathscr{S}(\varphi)$ is the so-called action of the transition. This means that for large enough values of $\Omega$, the stochastic transition will concentrate along the path, $\varphi^{*}$, which minimizes the action:

$$
\mathcal{S} \equiv \mathscr{S}\left(\varphi^{*}\right)=\min _{\varphi} \mathscr{S}(\varphi) .
$$

For the $n$-dimensional stochastic differential equation $\dot{\varphi}=f(\varphi)+g(\varphi) \Omega^{-\frac{1}{2}} \xi(t)$, the action for any path $\varphi_{\tau}$ of duration $\tau$ is given by the Freidlin-Wentzell functional [32]:

$$
\mathscr{S}\left(\varphi_{\tau}\right)=\frac{1}{2} \int_{0}^{\tau}\left\|\dot{\varphi}_{\tau}(t)-f\left(\varphi_{\tau}(t)\right)\right\|_{g\left(\varphi_{\tau}(t)\right)}^{2} d t,
$$

where $f\left(\varphi_{\tau}\right)$ is the deterministic field, and the multiplicative noise appears in the norm $\|\bullet\|_{g\left(\varphi_{\tau}\right)}^{2}$, corresponding with the inner product $\left\langle\bullet,\left(g\left(\varphi_{\tau}\right) g\left(\varphi_{\tau}\right)^{\top}\right)^{-1} \bullet\right\rangle$, where $g\left(\varphi_{\tau}\right) g^{\top}\left(\varphi_{\tau}\right) \equiv D$ is the diffusion matrix. In the Hopf model, $D$ takes the form 


$$
D(x, y)=\left(\begin{array}{cc}
\rho_{x}^{+}+\rho_{x}^{-} & 0 \\
0 & \rho_{y}^{+}+\rho_{y}^{-}
\end{array}\right) .
$$

Interestingly, the action and, consequently, the most probable path, are independent of $\Omega$. Additionally, the mean first passage time $T$ from one attractor to the other can be expressed as [32,39]

$$
T \simeq C e^{\Omega \mathcal{S}} .
$$

In order to numerically find the path minimizing $\mathscr{S}\left(\varphi_{\tau}\right)$, each path of duration $\tau$ was divided into a chain of $N$ segments with initial and final points in the relevant attractors. This reduces finding the optimal path to a minimization problem with $2(N-2)$ degrees of freedom. This was solved using the Broyden-Fletcher-GoldfarbShanno algorithm [40,41], using the analytical expression for the gradient of the action in any of the $2(N-2)$ dimensions [42].

Results.-To assess whether MAP theory can characterize the escape from a stable limit cycle, we have divided the analysis into two sections. First, we compare the MAP with paths obtained numerically from the Master Equation and the CLE. We then compare MAP predictions of the MFPT with those derived from CLE numerical solutions.

The MAP predicts average stochastic escape trajectories.-The MAP defines the most probable transient during the escape from the stable limit cycle at low noise. Direct comparison of the MAP with trajectories obtained from numerical integration of the CLE or Gillespie simulations shows good agreement (Figs. 1 and 2). As $\Omega$ increases, the stochastic escape trajectories converge to the MAP. In addition, our simulations show that the MAP recapitulates changes in parameters, such as the angular velocity of the Hopf model $\omega$ (Fig. 1), and it works well in cases with very asymmetrical basins of attraction as the Morris-Lecar model (Fig. 2 and Fig. S2 in Supplemental Material [35]). A very important feature of the MAP method is that it is much faster than regular CLE for the relevant range of $\Omega$. Additionally, the prediction becomes more precise the larger $\Omega$ is without an increase in computational time, while the CLE transition simulation time increases exponentially with $\Omega$ (for more details see Sec. III and Fig. S3 in Supplemental Material [35]).

A more detailed comparison between stochastic simulations and MAP theory reveals that the prediction of the latter becomes less accurate close to the exit point from the cycle. The small discrepancy originates in a fraction of trajectories following the limit cycle for a bit longer before starting the transition (Fig. 1). This results in the prediction of a smaller exit angle than the actual average exit angle (Figs. 2 and 3). Strikingly, this discrepancy was smaller than other methods such as using the probability distribution along the cycle, or the probability of escape from a thin annulus around the limit cycle (Figs. 2 and 3). The latter has

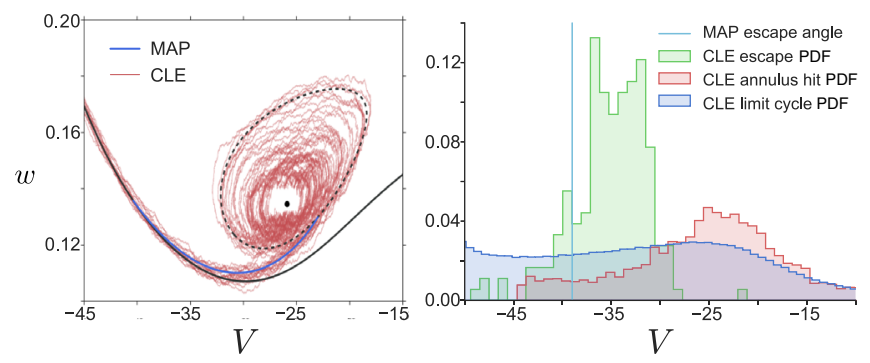

FIG. 2. Comparison of escape trajectories of the Morris-Lecar model limit cycle. (Left) MAP prediction (blue) compared with 30 CLE trajectories (red) $\Omega=10^{4}$. For the sake of clarity, only the last fraction of each trajectory prior to escape is shown. (Right) Comparison of the escape voltage distribution from the limit cycle for $\Omega=20000$ (green) with MAP prediction (cyan line). The results are also compared with the distribution of escape values of $V$ from a distance $\Delta w=0.0005$ from the limit cycle (red), and the occupation distribution along the limit cycle, i.e., before escape, (dark blue).

been proposed in Ref. [43] as a quantity that characterizes escape from a stable limit cycle in the low noise limit. The MAP proves to be the most useful of the three because action minimization takes place along the whole escape trajectory, instead of relying on a purely local analysis.

Localized inaccuracies in the MAP prediction suggest a highly heterogeneous contribution to the action along the MAP. In order to study this, we have evaluated the density of the action along the MAP, i.e., the Lagrangian of the system. Results show that the density is highest in the middle of the MAP (Fig. 3), while the density is negligible close to the stable and unstable limit cycles, where, in addition, the MAP is tangent to both limit cycles. This

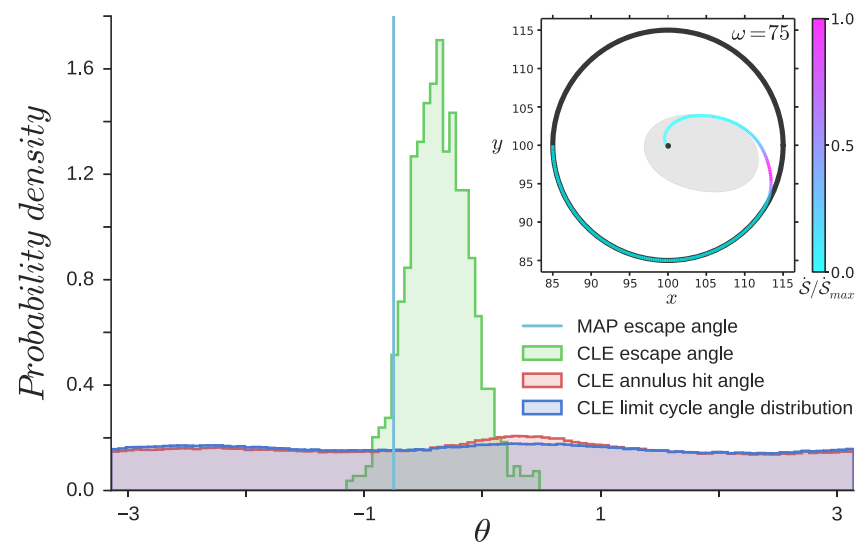

FIG. 3. Comparison of the escape angle distribution from the limit cycle (green) with the escape angle predicted by the MAP (cyan line) for $\omega=75$ and $\Omega=150$ in the Hopf model. The results are also compared with the distribution of escape angles from an annulus of radius 0.001 around the limit cycle (red), and the angular distribution along the limit cycle, i.e., before escape, (dark blue). Inset: Action density (Lagrangian) along MAP normalized to the maximum density. Other parameters are the same as in Fig. 1. 
suggests that there might be multiple paths with similar actions close to the exit point, giving an explanation for the discrepancy in the exit point from the cycle.

In usual escape problems, the path crosses from one basin of attraction to the other at the saddle point of the deterministic system. Here the boundary between basins of attraction is the unstable limit cycle, so the crossing point cannot be identified by a simple local stability analysis. This again shows the predictive power of the MAP approach.

Minimum action theory predicts MFPT for escape from the cycle.-To test the ability of MAP theory to predict the MFPT to exit the basin of attraction, we consider Eq. (6), which shows that this can be achieved to logarithmic precision, up to a constant, $C$. When the basins of attraction are separated by a saddle point, $C$ can be determined by a Jacobian computed at the saddle [44]. However, in the current case, the separatrix is an unstable limit cycle and $C$ must be computed numerically by solving the CLE at low $\Omega$. Our results show that the minimum action theory allows us to capture the MFPT dependence on model parameters (Fig. 4). In particular, we observe an increase in the MFPT with $\omega$. In fact, $C$ also depends nonmonotonically on $\omega$ (see Fig. 4). Nevertheless, as $\Omega$ grows, the contribution of the prefactor becomes less important $(\ln T \approx \Omega S+\ln C)$, and the minimum action dominates the escape time estimate.

In addition to the MFPT, we are interested in finding the probability distribution of escape times from the stable
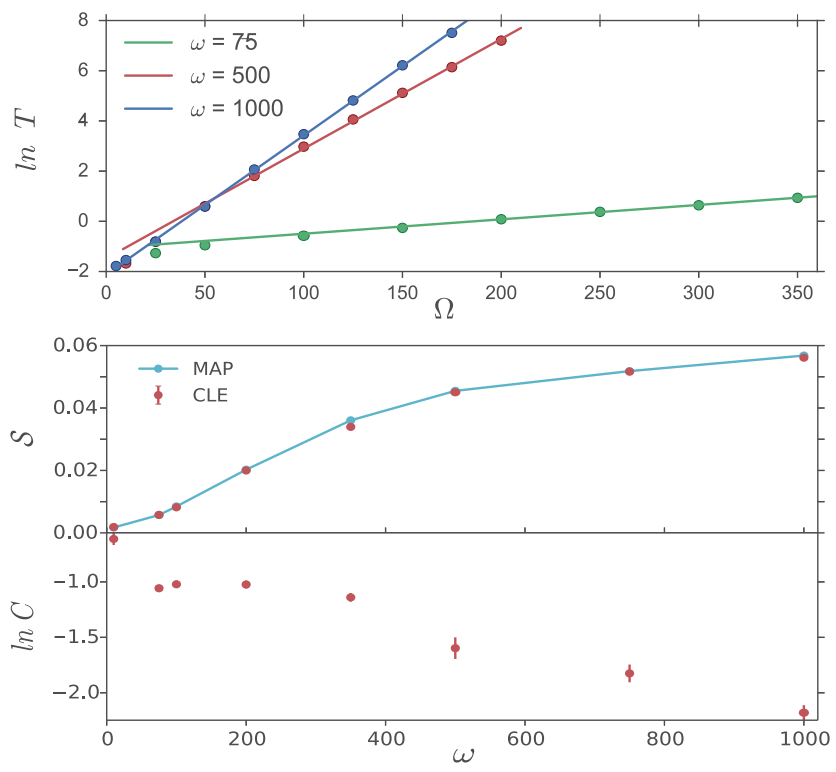

FIG. 4. Comparison of predictions of the MFPT for the Hopf model. (Top) Comparison of MFPTs calculated from CLE simulations (circles) with the exponential dependence of the MFPT on $\Omega$ given by $\mathcal{S}$ (lines). Each line is computed by minimizing the action $\mathcal{S}$ for different $\omega$ and fitting the prefactor $C$. (Bottom) Following the same procedure, the values of the action $\mathcal{S}$ and $C$ are compared for different values of $\omega$. Parameters values are the same as those of Fig. 1, error bars are standard error of the mean from the CLE. limit cycle. Assuming that escape is a rare event focused around a certain point in the cycle, the escape problem can be described as a Bernoulli process with low success probability $p$ taking place every period of the cycle $\tau=2 \pi / \omega$, at times $t_{n}=2 \pi n / \omega$. The probability of exiting at the $n$th revolution follows the geometric distribution $\mathcal{P}\left(t_{n}\right)=p(1-p)^{\left(\omega t_{n} / 2 \pi\right)-1}$. Following rare event theory, we can write the success probability as $p=e^{-\mathcal{S} \Omega} / C$, obtaining the geometric distribution,

$$
\mathcal{P}\left(t_{n}\right)=\frac{\left(1-e^{-\mathcal{S} \Omega} / C\right)^{\frac{\omega t_{n}}{2 \pi}}}{C e^{\mathcal{S} \Omega}-1} .
$$

In the limit $p \rightarrow 0$, the discrete geometric distribution Eq. (7) can be approximated by its continuum counterpart, the exponential distribution, which does not depend explicitly on the angular velocity,

$$
\mathcal{P}(t)=\frac{1}{C} e^{-\mathcal{S} \Omega+\frac{t}{C} \exp (-\mathcal{S} \Omega)} .
$$

Comparing the distributions Eqs. (7) and (8), with the probability distribution of MFPT obtained over several CLE realizations of the Hopf model, we obtained a good agreement (see Fig. 5). Surprisingly, even for realizations
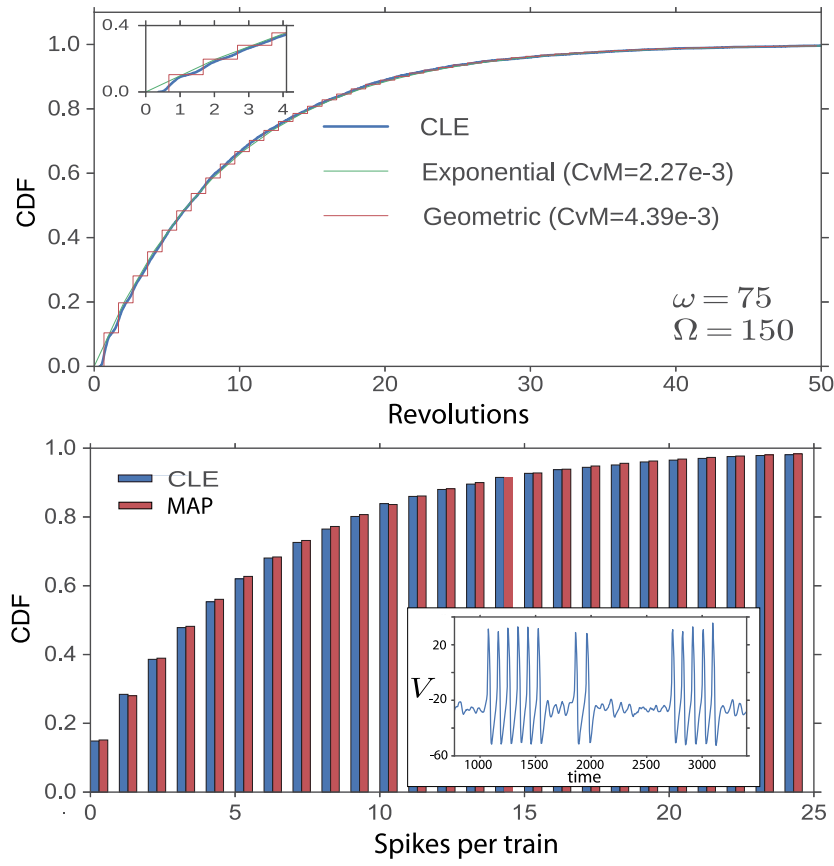

FIG. 5. (Top) Comparison of the cumulative distribution function (CDF) of the number of turns $t /(2 \pi \omega)$ for 10000 realizations of the Hopf model with $\omega=75$. For each distribution the Cramer-von Mises ( $\mathrm{CvM}$ ) criterion is computed to compare the resulting distribution with the geometric and exponential distribution determined by the value of the action. (Inset) Detail of the CDF for small revolution numbers. (Bottom) $\mathrm{CDF}$ of the number of spikes per train for the Morris-Lecar model comparing a CLE simulation of a total time 2100000 , with the geometric distribution predicted by the MAP method. (Inset) Trajectory exhibiting spike trains. 
with a low average number of revolutions prior to escape, the resulting probability distribution is more similar to an exponential distribution than to a geometric one. This is true even for escapes that occur during the first revolution, suggesting that $\theta$ differs significantly from $\omega t$. A more accurate prediction would involve a convolution of geometric processes with the angular noise $[45,46]$. However, for the parameters we used, the exponential distribution fits well independently of the average number of revolutions. Fitting the distribution Eq. (8) to the MPFTs from CLE realizations therefore provides an alternative method to compute the prefactor $C$. Similarly, the MAP is able to capture the distribution of the number of spikes in the neuronal activation bursts of the Morris-Lecar model. Each spike of a train will correspond with a whole turn around the limit cycle before switching to the rest state (stable node). Strikingly, the predicted geometric distribution resulting from the action, fits the burst distribution even when the number of spikes is small (Fig. 5).

Conclusions and perspectives.-We have shown that, within the rare event theory framework, escape problems from a stable limit cycle can be accurately characterized. The success of the method, in comparison with alternatives, relies on the fact that the Freidlin-Wentzell action is not a local property of the dynamical landscape but of the whole escape trajectory. For sufficiently large system sizes, we have shown that MAP theory accurately predicts escape trajectories and the escape time distribution. The method has also revealed properties of the escape trajectory, such as the tangent exit of the MAP from the stable limit cycle and tangent entry into the basin of attraction of the stable fixed point, as well as the dependence of the entry and exit points on the parameters of the system. In addition to its predictive power, the MAP method has also proven to be computationally faster than running Langevin simulations to study basin of attraction transitions whose simulation times increase exponentially with the size of the system.

MAP predictions, while better than previously used methods, were less successful in determining the exit angle from the stable limit cycle. A deeper analysis of the exit angle could be carried out through a relaxation of the Laplace condition, i.e., the reduction to an integral along a single optimal path, by further exploring the distribution of suboptimal paths. Additionally, in the absence of a unique point (the saddle) separating the basins of attraction, novel research into the calculation of the prefactor [44] should be extended.

R. dIC. acknowledges AGAUR for funding under its doctoral scholarship program and Fundació Ferran Sunyer i Balaguer. R. dlC. and T. A. are supported by the CERCA Program of the Generalitat de Catalunya, MINECO (Grants No. MTM2015-71509-C2-1-R and No. CRM-BGSMath "María de Maeztu" program MDM-2014-0445), and AGAUR (Grant No. 2014SGR1307). R. P-C., P. G. and
K. M.P. were supported by the Wellcome Trust (Grant No. WT098325MA).

R. dlC. and R. P-C. contributed equally to this work.

*Corresponding author.

r.carrasco@ucl.ac.uk

[1] T. B. Kepler and T. C. Elston, Biophys. J. 81, 3116 (2001).

[2] M. Kaern, T. C. Elston, W. J. Blake, and J. J. Collins, Nat. Rev. Genet. 6, 451 (2005).

[3] N. Maheshri and E. K. O'Shea, Annu. Rev. Biophys. Biomol. Struct. 36, 413 (2007).

[4] R. Losick and C. Desplan, Science 320, 65 (2008).

[5] A. Eldar and M. B. Elowitz, Nature (London) 467, 167 (2010).

[6] P. Guerrero, H. M. Byrne, P. K. Maini, and T. Alarcón, J. Math. Biol. 72, 123 (2016).

[7] B. D. MacArthur, C. P. Please, and R. O. C. Oreffo, PLoS One 3, e3086 (2008).

[8] G. Balazsi, A. van Oudenaarden, and J. J. Collins, Cell 144, 910 (2011).

[9] C. Gonzalez, J. C. J. Ray, M. Manhart, R. M. Adams, D. Nevozhay, A. V. Morozov, and G. Balazsi, Mol. Syst. Biol. 11, 827 (2015).

[10] J. DiStefano, Dynamic Systems Biology Modeling and Simulation (Elsevier Science, New York, 2015), ISBN 9780124104938.

[11] J. Jaeger and N. Monk, J. Physiol. 592, 2267 (2014).

[12] B. Verd, A. Crombach, and J. Jaeger, BMC Syst. Biol. 8, 43 (2014).

[13] A. Goldbeter, Proc. Natl. Acad. Sci. U.S.A. 261, 319 (1995).

[14] J.-C. Leloup and A. Goldbeter, BioEssays 22, 84 (2000).

[15] P. Smolen, D. A. Baxter, and J. H. Byrne, J. Neurosci. 21, 6644 (2001).

[16] A. Goldbeter, Biochemical Oscillations and Cellular Rhythms: The Molecular Bases of Periodic and Chaotic Behaviour (Cambridge University Press, Cambridge, England, 1996).

[17] C. Gerard and A. Goldbeter, Proc. Natl. Acad. Sci. U.S.A. 106, 21643 (2009).

[18] C. Gerard and A. Goldbeter, Interface Focus 1, 24 (2011).

[19] C. Gerard and A. Goldbeter, Frontiers in Physiol. 3, 413 (2012).

[20] K. Tsumoto, H. Kitajima, T. Yoshinaga, K. Aihara, and H. Kawakami, Neurocomputing; Variable Star Bulletin 69, 293 (2006).

[21] S. Ditlevsen and P. Greenwood, J. Math. Biol. 67, 239 (2013).

[22] K. Bodova, D. Paydarfar, and D. B. Forger, J. Theor. Biol. 365, 40 (2015).

[23] C. Morris and H. Lecar, Biophys. J. 35, 193 (1981).

[24] T. Tateno and K. Pakdaman, Chaos 14, 511 (2004).

[25] D. F. Anderson and T. G. Kurtz, Stochastic Analysis of Biochemical Systems (Springer, New York, NY, 2015).

[26] D. T. Gillespie, J. Phys. Chem. 81, 2340 (1977).

[27] Y. Cao, D. T. Gillespie, and L. R. Petzold, J. Comp. Physiol. 206, 395 (2005).

[28] P. Hänggi, P. Talkner, and M. Borkovec, Rev. Mod. Phys. 62, 251 (1990).

[29] C. W. Gardiner, J. Stat. Phys. 30, 157 (1983). 
[30] M. I. Dykman, E. Mori, J. Ross, and P. M. Hunt, J. Chem. Phys. 100, 5735 (1994).

[31] R. S. Maier and D. L. Stein, J. Stat. Phys. 83, 291 (1996).

[32] M. I. Freidlin and A. D. Wentzell, Random Perturbations of Dynamical Systems (Springer, New York, 1998).

[33] E. Weinan, W. Ren, and E. Vanden-Eijnden, Commun. Pure Appl. Math. 57, 637 (2004).

[34] Y. A. Kuznetsov, Elements of Applied Bifurcation Theory (Springer, New York, 2014), ISBN 1475724225.

[35] See Supplemental Material at http://link.aps.org/ supplemental/10.1103/PhysRevLett.120.128102 for more details on the models and its stochastic derivation.

[36] H. A. Brooks and P. C. Bressloff, Phys. Rev. E 92, 012704 (2015).

[37] N. van Kampen, Stochastic Processes in Physics and Chemistry (Elsevier, New York, 2007), ISBN 978-0-444-52965-7.
[38] D. T. Gillespie, J. Chem. Phys. 113, 297 (2000).

[39] H. Touchette, Phys. Rep. 478, 1 (2009).

[40] R. Fletcher, Practical Methods of Optimization (Wiley, New York, 2000), ISBN 0471494631.

[41] GNU Scientific Library Reference Manual (Network Theory Ltd., 2009), 3rd ed., ISBN 0954612078, https:// www.gnu.org/software/gsl/.

[42] R. Perez-Carrasco, P. Guerrero, J. Briscoe, and K. M. Page, PLoS Comput. Biol. 12, e1005154 (2016).

[43] P. Hitczenko and G. S. Medvedev, J. Nonlinear Sci. 23, 835 (2013).

[44] F. Bouchet and J. Reygner, Ann. Henri Poincare 17, 3499 (2016).

[45] N. Berglund and B. Gentz, SIAM J. Math. Anal. 46, 310 (2014).

[46] N. Berglund, Markov Proc. Relat. Fields 22, 467 (2016). 